\title{
Optical sensor array platform based on polymer electronic devices
}

\author{
Marc M. Koetse $^{a}$, Peter A. Rensing ${ }^{a}$, Ruben B. A. Sharpe ${ }^{a}$, Gert T. van Heck ${ }^{a}$, Bart A. M. \\ Allard $^{a}$, Nicole N. M. M. Meulendijks ${ }^{b}$, Peter G. M. Kruijt ${ }^{a}$, Marcel W. W. J. Tijdink ${ }^{a}$, René \\ M. de Zwart ${ }^{b}$, René J. Houben ${ }^{b}$, Eric Enting ${ }^{b}$, Sjaak J. J. F. van Veen ${ }^{c}$, Herman F. M. Schoo ${ }^{a}$ \\ ${ }^{a}$ Holst Centre / TNO, P.O. Box 8850, 5605 KN Eindhoven, the Netherlands; \\ ${ }^{b}$ TNO Science \& Industry, P.O. Box 6235, 5600 HE Eindhoven, the Netherlands; \\ ${ }^{c}$ TNO Quality of Life, P.O. Box 360, 3700 AJ Zeist, the Netherlands
}

\begin{abstract}
Monitoring of personal wellbeing and optimizing human performance are areas where sensors have only begun to be used. One of the reasons for this is the specific demands that these application areas put on the underlying technology and system properties. In many cases these sensors will be integrated in clothing, be worn on the skin, or may even be placed inside the body. This implies that flexibility and wearability of the systems is essential for their success. Devices based on polymer semiconductors allow for these demands since they can be fabricated with thin film technology. The use of thin film device technology allows for the fabrication of very thin sensors (e.g. integrated in food product packaging), flexible or bendable sensors in wearables, large area/distributed sensors, and intrinsically low-cost applications in disposable products. With thin film device technology a high level of integration can be achieved with parts that analyze signals, process and store data, and interact over a network. Integration of all these functions will inherently lead to better cost/performance ratios, especially if printing and other standard polymer technology such as high precision moulding is applied for the fabrication. In this paper we present an optical transmission sensor array based on polymer semiconductor devices made by thin film technology. The organic devices, light emitting diodes, photodiodes and selective medium chip, are integrated with classic electronic components. Together they form a versatile sensor platform that allows for the quantitative measurement of 100 channels and communicates wireless with a computer. The emphasis is given to the sensor principle, the design, fabrication technology and integration of the thin film devices.
\end{abstract}

Keywords: OLED, optical sensor, opto-electronic polymers, printing, reel-to-reel

\section{INTRODUCTION}

Sensing systems for defense and security mostly focus on early identification of threats. In a time in which terrorist attacks are perceived as an increasing threat, it is, obviously, very important to be able to detect chemical markers that reveal the presence of hidden explosives, or to quickly identify the nature of pathogens making up a biological attack. Likewise, on the battlefield, it is important to be able to "sniff out" the location of landmines, or to keep track of enemy troop movements using, e.g. seismic sensors.

Another class of battlefield usage altogether is that for optimizing medical treatment in order to increase survivability. The most prevalent battlefield injuries, some $70 \%$, are to the upper and lower extremities. ${ }^{?}$ This percentage has been consistent since world war 2 . Such injuries are fortunately not instantly fatal; they are, however, very susceptible to sepsis, specifically when they pertain to burn wounds. It is, therefore, imperative to develop systems that minimize the need for changing of the wound dressing in the field, in order to minimize exposure to ambient pathogens. Such systems call for the ability to monitor, e.g. both bacterial growth and vascular regeneration in the wound area. Typically, this requires large area sensors to enable monitoring of the whole wound area, and flexible sensors to enable conformal contact enable integration in bandage and to minimize patient discomfort.

Correspondence should be addressed to M.M.K.: marc.koetse@tno.nl

Electro-Optical Remote Sensing, Detection, and Photonic Technologies and their Applications edited by G. W. Kamerman, O. K. Steinvall, K. L. Lewis, K. A. Krapels, J. C. Carrano, A. Zukauskas Proc. of SPIE Vol. 6739, 67391D, (2007) · 0277-786X/07/\$18 - doi: 10.1117/12.737265

Proc. of SPIE Vol. 6739 67391D-1 
In this paper, a sensor platform will be presented that enables large area sensing of multiple markers. The platform can be built on flexible substrates, which eventually should enable integration of the sensor-system with the wound dressing. Moreover, since it is intended as a throw-away application, special attention will be paid to cost efficient manufacturing. An elementary approach to the reduction of production costs is standardization. Standard components are likely to be less expensive than customized components, because they will be produced in higher volume. Higher volume production allows greater economies of scale and more learning, and is more likely to attract competitors who exert price pressure on one another. ${ }^{1}$ Another key element is the reduction of materials consumption. Specifically for fine chemicals, the materials costs may run so high that they end up constituting $\gg 85 \%$ of the total running costs. This is exemplified below in Section 2.1. In this paper, thus, the guiding principles toward cost efficient manufacturing that are used for the sensor design are:

- Standardization: Use of a generic platform, which can be adapted to specific sensing tasks. Standardization here also pertains to 'component' standardization.

- Materials consumption: Use additive technology (as opposed to subtractive), and apply material only were needed.

- High volume processing: Flexible substrates, allowing reel-to-reel, continuous, processing as well as integration in bandaging material.

Although the choices above are in principle aimed at cost efficient production technology for a generic sensing platform, they bare certain advantages for specific sensing applications. For example, the use of flexible substrates allows very simple solutions for sensing problems that require conformal contact of the sensor with a curved surface or a surface with a dynamic topography, over larger surface areas. Such applications can for example be found in the areas of smart textiles and wearable monitoring systems. ${ }^{2,3}$ Also, the use of additive (printing) technology facilitates the inclusion of multiple functionalities on a single substrate (because of the large control over the local materials composition) and makes this virtually independent of the substrate's size. In the following, such a sensor platform will be described in more detail.

\section{SENSING SCHEME}

In a sensor design, several components can be distinguished by their functionality. The sensor proper is the component that translates the stimulus that is to be detected (e.g. a chemical compound or a physical property such as temperature or acceleration) into a signal that can be measured, i.e. typically an electrical signal. There are many things that can be sensed, and therefore there are many sensing schemes. Apart from the sensor proper there are supporting components, such as an energy supply and perhaps some logic for low level signal manipulation. The discussion in this paper is limited to the sensor proper in a sensing scheme that relies for its signal transduction on an induced change in the optical path between a light source and a photodiode. In principle, this is a multipurpose sensing scheme that allows sensing of various stimuli. It can be adapted for a specific sensing application merely by modifying the component between the light source and the photodiode. The light source and the photodiode in principle remain unaffected by such modification and, therefore, can be standardized. Importantly, the form of the output, which is an electrical signal, is invariant with respect to the form of the input. This allows for all downstream processing elements to also be standardized. A schematic of such scheme is depicted in Figure 1. The multifunctionality of such platform will be discussed in Section 3

For the application of the sensor as a monitoring device for wound healing, the sensor is designed with the light source and the photodiode in a single plane. The responsive element, i.e. the medium that is responsible for the change in the properties of the emitted light, is the wound itself. Light emitted from the light source will scatter diffusely in the wound area and, upon interacting with the there present elements (e.g. serum, red blood cells or bacteria), part of the light will be scattered back to the photodiode (Figure 2). The functional components of such in-plane optical sensor platform (IPOS), thus, are the light source, the responsive element and the light detection component, which can be constructed as independent modules. For reasons of intrinsic printability, the light source is chosen to be an organic light emitting diode (OLED) and the detector is an organic photovoltaic device (PVD). These will be discussed in the following.

Proc. of SPIE Vol. 6739 67391D-2 


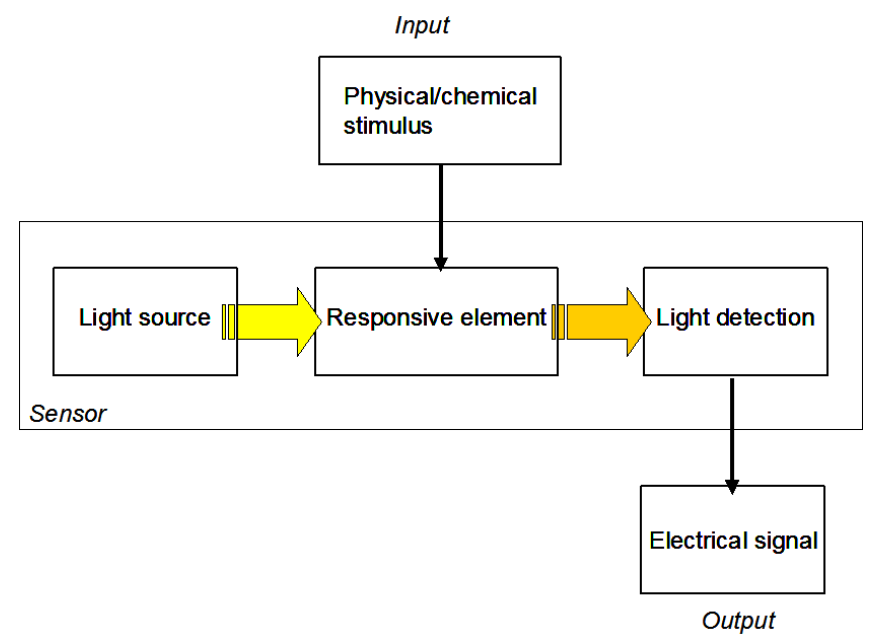

Figure 1. Schematic representation of a generic optical sensing scheme. A change in input stimulus requires a change in the responsive element but not of the other components.

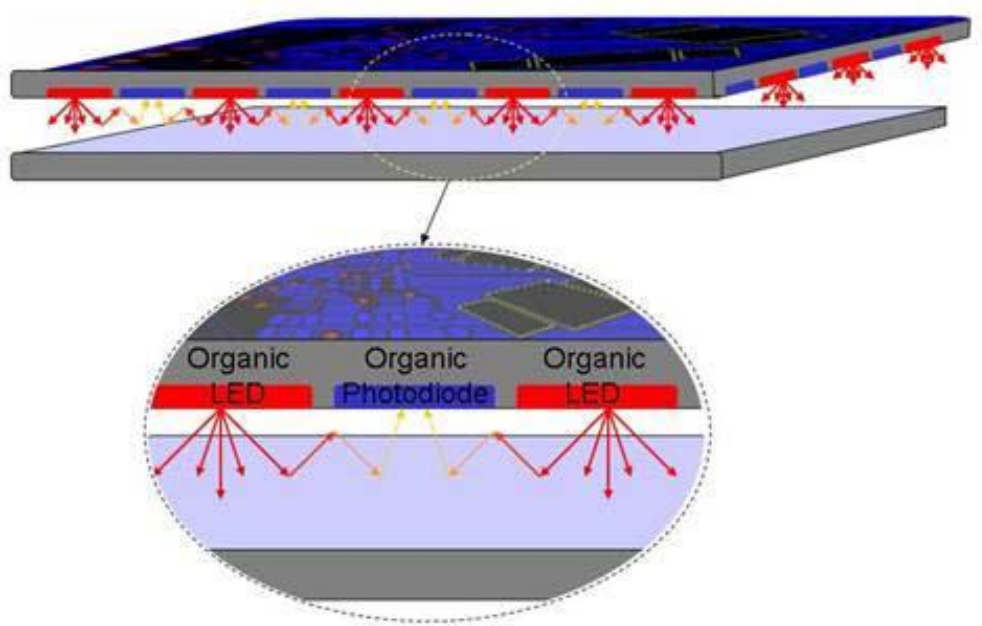

Figure 2. Schematic representation of an in-plane optical sensor platform. Organic LED and organic PVD cells are produced in the same plane, on top of a flexible foil, onto which a film of flexible (organic) logic for basic processing is laminated. Light emitted from the LEDs is scattered back from the area of interest (in this paper the wound area) and detected by the photodiodes that are located next to the LEDs.

\subsection{OLED Module}

\subsubsection{Materials consumption}

An OLED device in its simplest form consists of conjugated organic molecules with a suitable bandgap, sandwiched between electrodes with matching workfunctions, at least one of which is transparent. Additional elements may be added to enhance the performance and the device lifetime. Typically, to this end, at least a poly(3,4ethylenedioxythiophene)/polystyrene sulfonic acid (PEDOT/PSS) hole injection layer is employed. The organic materials in an OLED device are expensive and contribute significantly to the overall costs. This may be exemplified in a model calculation showing an estimate of the cost distribution associated with the coating of the two organic layers in a simple OLED device, namely PEDOT/PSS and light emitting polymer (LEP), on a polyethylene naphthalate (PEN) substrate with a pre-deposited indium-tin-oxide (ITO) anode. Several process steps are considered (listed on the independent axis of the diagram in Figure 3 ) and a production velocity of $33 \mathrm{~m} / \mathrm{min}$, a 


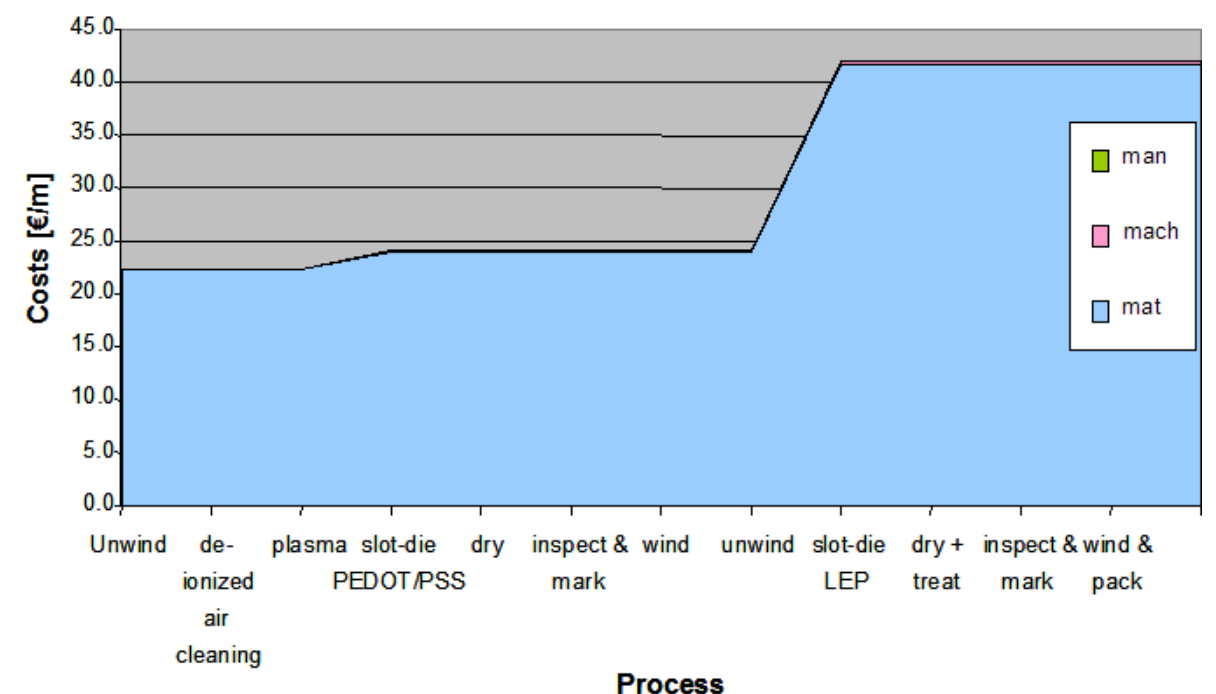

Figure 3. Cumulative cost diagram, in $\mathrm{M} € / \mathrm{m}$, of the process for applying PEDOT/PSS and LEP on a foil. The process steps are listed from left to right on the independent axis. The cost that is plotted for one process step, includes the costs incurred from all the previous steps. Contributing to the total cost are the materials cost (mat), the machine depreciation (mach) and personnel (man). The contribution of the latter two can hardly be distinguished in the diagram.

Table 1. Cost distribution of the process for applying PEDOT/PSS and LEP on a foil.

\begin{tabular}{|c|c|c|}
\hline materials $[\mathrm{M} € / \mathrm{m}]$ & machine depreciation $[\mathrm{M} € / \mathrm{m}]$ & manpower $[\mathrm{M} € / \mathrm{m}]$ \\
\hline 41.67 & 0.48 & 0.05 \\
\hline \hline \multicolumn{3}{|c|}{ Total cost $=42.20 \mathrm{M} € / \mathrm{m}$} \\
\hline
\end{tabular}

yield of $70 \%$, a single shift manning the process line and an initial investment of $7 \mathrm{M} €$ with a depreciation time of 5 years are assumed. From this 'model' an estimation can be made of the cumulative costs that are incurred for each process step (Figure 3). The conclusion from such an exercise is that for organic electronics, which require very expensive conductive organic materials, the materials consumption may constitute as much as up to $99 \%$ of the total running costs (Table 1). Even in a best case scenario, lowering the cost of the LEP material to that of PEDOT/PSS (which may be feasible for a mature production process) and an extra investment of $8 \mathrm{M} €$, the materials consumption may still account for $>85 \%$ of the running costs.

Clearly, wasteful processes such as spincoating, which suffice in the lab, are not compatible with cost efficient manufacturing of OLED-based devices. Printing is a deposition technology that produces relatively little waste and is therefore a good candidate for application in OLED manufacturing. The printing of OLEDs has been well researched in the past, the main aim being the production of displays. ${ }^{4}$ There exist several printing technologies that can in principle be employed for OLED device manufacturing. The size of a pixel in a display is of the order of a few hundred micrometer; these can conveniently be printed using inkjet. Depending on the application, a single OLED pixel in a sensor device may vary considerably. To this end other printing technologies are to be investigated such as such as relief printing, flexographic printing or screenprinting. Their application is, however, not straightforward. Specifically the contact printing techniques such as intaglio and flexo, in which the printing equipment also contacts the areas of the substrate were no ink transfer is intended, must be considered with care.

\subsubsection{Thin-film encapsulation}

Reel-to-reel processing of OLEDs requires flexible substrates, which brings its own problems. An important problem that occurs with processing on polymeric substrates is that they are typically permeable for oxygen 


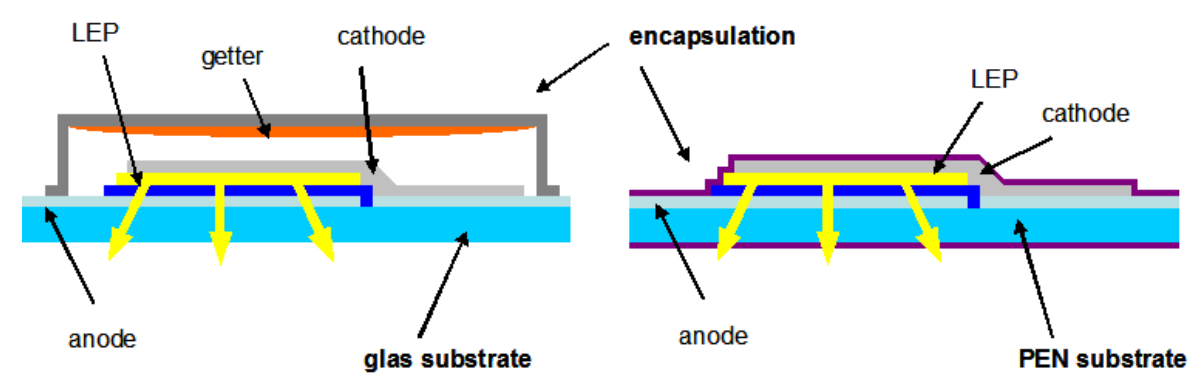

Figure 4. Schematic representation of a typical OLED device. The left scheme shows a classical OLED device, processed on glass and encapsulated together with a getter using a metal cap. The right scheme shows a similar device, but using thin-film encapsulation.
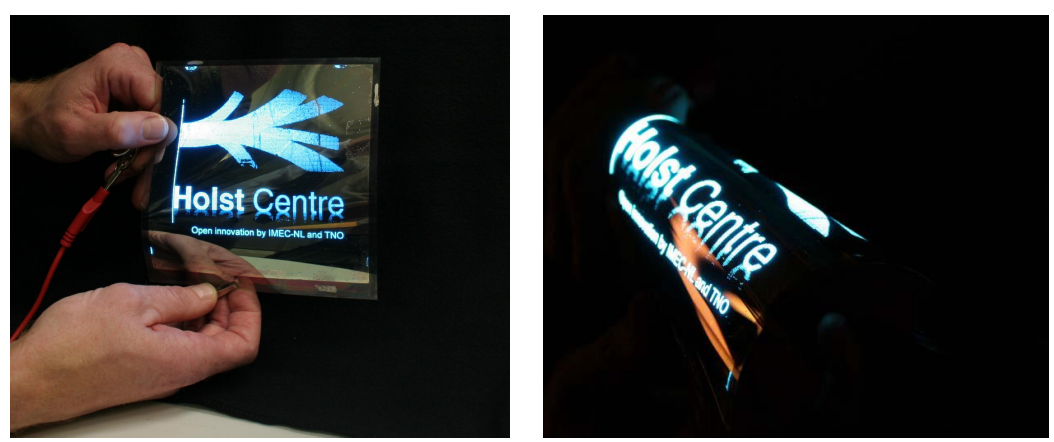

Figure 5. Photographs of a large area OLED device showing the Holst Centre logo. Clearly, the encapsulation allows light to leave the device and affects the flexibility only marginally, if noticeable at all.

and water. Since the workfunction of the cathode must be matched with the conduction band of the LEP, these materials are very reactive towards oxidation and are therefore sensitive to both oxygen and water. Classically, this problem is circumvented by processing on glass, which is not permeable, and by encapsulating the OLEDstack together with a getter with a metal cap (Figure 4). Processing on glas, however, is not compatible with reel-to-reel processing and encapsulating with a metal lid would not only be insufficient, but undesirable because of its implied loss of flexibility. To this end, several schemes toward thin-film encapsulation are being investigated (Figure 4).

As stated above, encapsulation serves to protect the cathode. This means that an OLED without encapsulation will work initially, but will deteriorate rapidly. The better the encapsulation, the longer the device will function. A single defect, however, will progressively affect the cathode, which in time will cause device failure. There are, therefore, currently two main approaches to thin film encapsulation. The first aims at creating perfect barriers and allows to this end significant redundancy. The second aims at delaying the debilitating effects of small defects, e.g. by increasing the diffusion path to the cathode and/or by inclusion of getters in the barrier. Both approaches have their merits as well as their faults. Apart from their functionality as barrier, the thin films should also be transparant in order to allow light to escape the device and it is undesirable for the barrier to much affect the flexibility of the device (Figure 5).

\subsection{PVD Module}

Efficient materials usage, being an argument for printing of the OLED devices, is an equally valid argument for printing of PVDs. Organic photodiodes, however, have mainly been researched for application in solar cells. These are typically large area applications, requiring high efficiencies. Surprisingly little attention has been given to the use of these devices as photodiodes. ${ }^{5-7}$ Rather than to the printability and sensitivity of small area devices, up to now the studies are directed towards increasing the efficiency through material research and device design. ${ }^{8-11}$ 


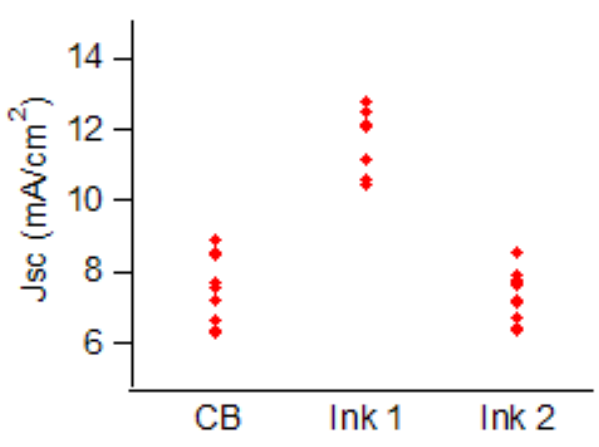

(a)

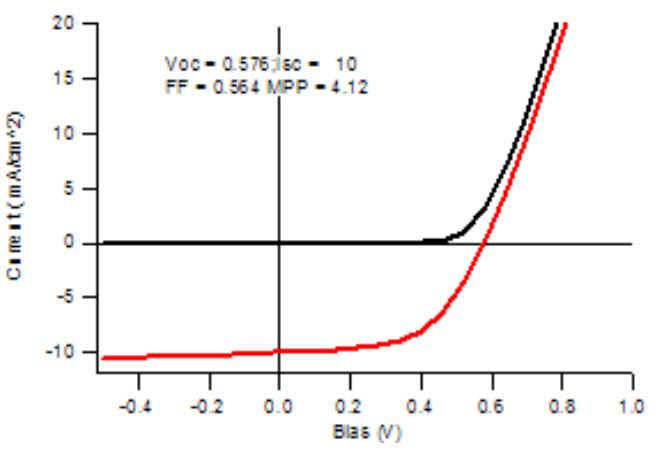

(b)

Figure 6. a) Photocurrents obtained from two formulations compared to "standard" chlorobenzene. b) IV curve of a device spin coated from Ink 1.

In this study, therefore, attention was paid to formulation of printable PVDs for use as photo-detectors. The active material was chosen to be a blend of poly(3-hexylthiophene) (P3HT) as donor and [6,6]-phenyl C60 butyric acid methyl ester (PCBM) as acceptor in a 1:1 ratio. This blend is known to be relatively forgiving with respect to layer thickness and morphology requirements, whilst still providing good photocurrents. The aim was to formulate this material for use in inkjet printing. In view of future high volume production, a complicating requirement, however, was the need to print from chlorine free solvents. Only solvents with high boiling points were screened, in order to avoid an annealing step that is not compatible with future co-application of OLEDs. This is an important consideration, because the printability of both PVDs and OLEDs allows their manufacture on a single substrate, which is of course imperative for the intended in-plane optical sensor platform.

First, a number of formulations were tested using spin coating as deposition method. Chlorobenzene (CB) was used as reference and the device structure was standard. ${ }^{9}$ Figure 6 a compares the photocurrent obtained from these devices spin coated from $\mathrm{CB}$ and two of the best formulations, labeled Ink 1 and Ink 2 respectively. Per formulation a total of eight devices were measured having active areas ranging from $1 \mathrm{~mm}^{2}$ to $10 \mathrm{~mm}^{2}$. Figure $6 \mathrm{~b}$ shows the IV curve of a device spin coated from Ink 1 with an area of $1.6 \mathrm{~mm}^{2}$. It should be noted that the devices were measured under a calibrated halogen lamp. The values may therefore be compared to each other but do certainly not reflect a measurement under AM1.5 conditions.*

The thickness of the active layers varied with formulation. Thicknesses of 165, 195, and $210 \mathrm{~nm}$ were respectively obtained when comparing CB-formulated ink with Ink 1 and Ink 2 (measured with a Veeco 'Dektak 8' profilometer). The improved performance of Ink 1 compared to CB (Figure 6) could partly be explained by the increased layer thickness. ${ }^{12}$ Additionally, the difference in performance may also be attributed to differences in the solvent evaporation rate. ${ }^{13}$

The high current, good open circuit voltage, and relatively good fill factor make that Ink 1 is chosen as the ink for inkjet print tests. The inkjet printed diodes were fabricated on patterned ITO/glass substrates. Again the device structure was standard and the active area was $2 \mathrm{~mm}^{2}$. Both PEDOT/PSS and Ink 1 were inkjet printed using a Dimatix 'DMP-2831' printer. Various printing strategies were used but were found not to have a significant impact on the performance of the devices. Figure 7 shows a typical I-V curve of such devices. The device performs less good than a spin coated one as indicated by the low fill factor. It is speculated that this originates in inhomogeneity of the layers. The current, however, is close to that found in the spin coated devices. Currently the printing strategy formulations are being optimized in order to improve these results.

Also, the photodetector was evaluated with respect to its response time for use in a pulse-oxymeter, which is a sensing device that can measure the hearth rate and the blood oxygenation level by monitoring the skin

* Solar Spectral Irradiance: Air Mass 1.5. The reference spectra of the American Society for Testing and Materials for the evaluation of photovoltaic performance. 


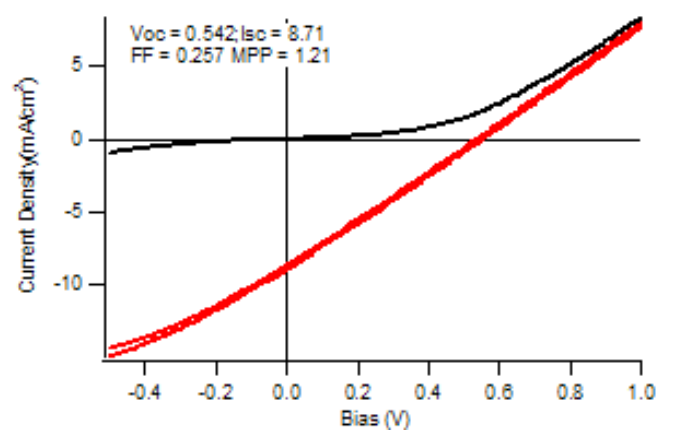

Figure 7. IV curve of a completely printed photodiode.

coloration. Using a red emitting led (Lumiled, which switches on in $\sim 20 \mathrm{~ns}$ ) a respectable response time was found of approximately $50 \mu \mathrm{s}$. This is more than sufficient for a measurement frequency of $3 \mathrm{kHz}$, which is the required frequency for use in the intened application.

\section{MULTIFUNCTIONALITY OF THE SENSOR PLATFORM}

The sensing concept relies on a change in the optical properties of a responsive material that is located between the light source and a detector. This change can be any one of several things. It can be a change in the transparency (opacity), a change in the transmittance (adsorption) or, with the aid of polarizing filters, even a change in polarization. Likewise, this change can be brought about in many different ways. There are whole classes of materials that change color upon a change in, e.g. temperature (thermochromic materials) or pressure (piezochromic materials). The inclusion of mechanical elements that modulate the progression of light can even extend the range of sensing applications to include e.g. accelerometry. ${ }^{14}$

Chemo-responsive materials form a very important class of responsive materials for use in this sensor concept. One only needs to refer to the large body of chemical analyses that are based on colorimetry or fluorescence to point out its relevance. Within the scope of an opto-chemical sensor platform, such as described in this paper, complex sensor combinations may be realized, adding extra functionality in one and the same platform, merely by redesigning the module of the responsive element. Sensor arrays may, e.g., include cells with thermochromic material so as to enable on-board thermal calibration. They may consist of multiple chemical specificities so as to enable parallel multi-analyte screening, or they may combine multiple detection mechanisms so as to achieve channel redundancy (detection of a single analyte using multiple independent detection methods). This, of course, calls for the ability to have local control over the materials composition of the array. Again, this is a feat that is enabled by printing.

In this study, the printing of coatings is investigated for the detection of ammonia. Responsive material was dissolved in an ethyl cellulose matrix, for the sake of uniformity in processing, to provide a carrier material and, because ethyl cellulose is relatively hydrophilic, to provide a stabile environment with respect to ambient humidity. Because of the requirements of the printing solution, namely with respect to viscosity; the volatility of the solvent; and the applied volume, inkjet printing unfortunately turned out to be ill suited to the task. Eventually, good results were obtained using a Lee company 'INKX0519550A' valve-jet that is capable of dispensing fluids in the $1 \mathrm{Pas}-35 \mathrm{Pas}$ range (Figure 8 ). The active material in the coating is bromothymol blue that has been made lipophilic by attachment of alkyl chains, in order to be able to dissolve it in the ethyl cellulose matrix. In spite of these alterations the responsive behavior remained very good (Figure 9).

Only a simple alteration is required to enable sensing in liquid environments. For this reason a closed cartridge was developed, which enables optical interrogation but optically isolates the different pixels. The design of the fluidic cartridge, however, is nontrivial, since flow and mixing effects may influence the sensor behavior (Figure 10). Although this is not investigated here, such a fluidic cartridge may be expanded with modules for sample pre-treatment, thereby enabling a (micro-) total analysis system $((\mu) \mathrm{TAS})$. 


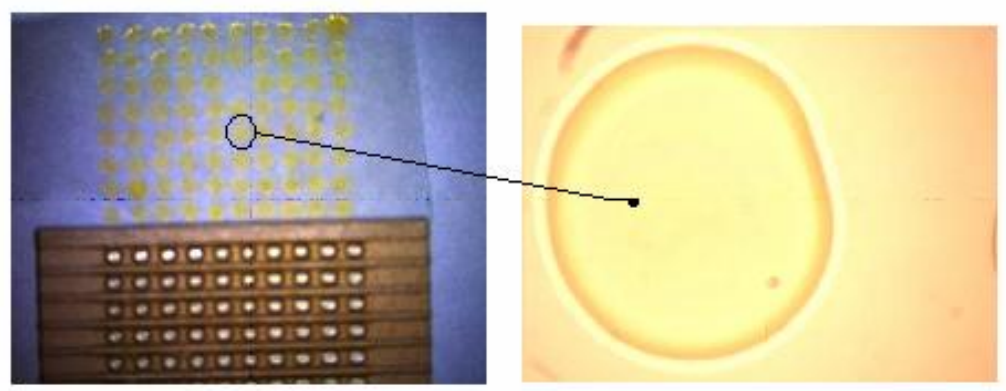

Figure 8. Image of valve-jetted responsive coating. The lower part of the left panel shows a carrier, which serves both as a substrate and to optically isolate the individual coating-dots. The right panel shows a blow-up of an individual dot.

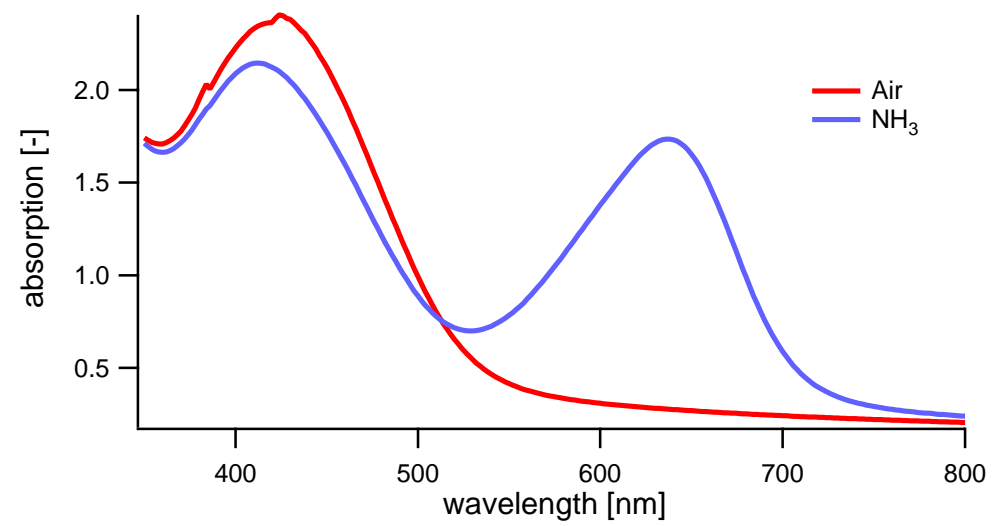

Figure 9. UV-VIS spectrum of an $\mathrm{NH}_{3}$-sensitive coating. Upon exposure to $\mathrm{NH}_{3}$, a clear increase in adsorption around $600 \mathrm{~nm}$ can be observed, which causes the coating to turn from yellow to blue.

\section{CONCLUSION}

In this paper a sensor principle is proposed that is based on an induced change in the optical path between an OLED and a PVD array. This design enables standardization, both on the platform and the component levels, because of standardization of the elements of the sensor proper as well as the downstream elements. On theoretic grounds this standardization is expected to support cost efficient manufacturing.

Other aspects of cost efficient manufacturing include materials waste reduction and high volume processing. Using printing technology to create the optically active elements is a promising route toward economical materials usage. In this paper, it is also remarked upon that printing technology offers advantages in terms of freedom of sensor design. It is shown that all the optical elements can indeed be printed. High volume processing is addressed by using flexible substrates. An important issue that arises from OLED processing on flexible substrates, however, is the need for very high quality thin-film encapsulation.

Because of the printability of the optical components, the OLED and the PVD array can, feasibly, be created in a single plane. This setup allows the future integration of the sensor system in wound dressing for the purpose of monitoring the healing (inflammation) processes. The printed PVD has been shown to meet the specifications for use in a pulse-oxymeter.

The versatility of the platform is frequently remarked upon in the text, and hinted at by showing how a simple adaption of the responsive module can prepare the sensor platform for sensing in liquid environments. A proper design of a total analysis system, however, requires more work. 

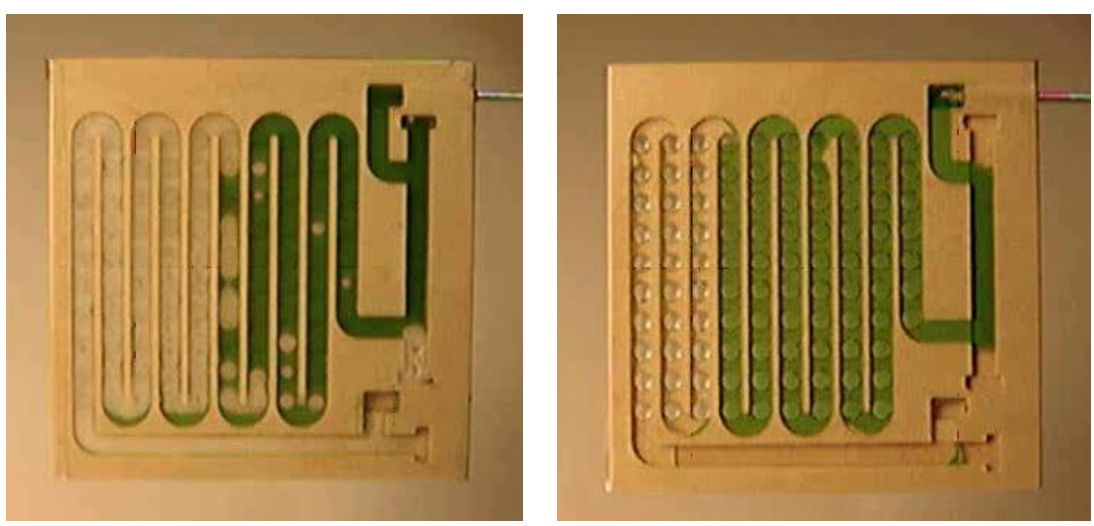

Figure 10. Photographs of a microfluidic array prototype. They show that with a proper design, the inclusion of air in the liquid stream as a result of turbulent flow, can be avoided. The left and right panels show identical meandering paths, with only a difference in the geometry of the matrix cells.

\section{REFERENCES}

1. K. Ulrich, "The role of product architecture in the manufacturing firm," Research Policy 24, pp. 419-440, 1995.

2. B. Gyselinckx, C. Van Hoof, J. Ryckaert, R. F. Yazicioglu, P. Fiorini, and V. Leonov, "Human++: Autonomous wireless sensors for body area networks," in Custom Integrated Circuits Conference, 2005. Proceedings of the IEEE, 2005.

3. S. Coyle, Y. Wu, K.-T. Lau, D. De Rossi, G. Wallace, and D. Diamond, "Technical feature - smart nanotextiles: A review of materials and applications," MRS Bulletin 32(5), p. 434, 2007.

4. N. C. Van der Vaart, H. Lifka, F. P. M. Budzelaar, J. E. J. M. Rubingh, J. J. L. Hoppenbrouwers, J. F. Dijksman, R. G. F. A. Verbeek, R. Van Woudenberg, F. J. Vossen, M. G. H. Hiddink, J. J. W. M. Rosink, T. N. M. Bernards, A. Giraldo, N. D. Young, D. A. Fish, M. J. Childs, W. A. Steer, D. Lee, and D. S. George, "Towards large-area full-color active-matrix printed polymer oled television," Journal of the Society for Information Display 13(1), pp. 9-16, 2005.

5. G. Yu, G. Srdanov, J. Wang, H. Wang, C. Y., and A. J. Heeger, "Large area, full-color, digital image sensors made with semiconducting polymers," Synth. Met. 111-112, pp. 133-137, 2000.

6. T. Someya, S. Iba, Y. Kato, T. Sekitani, Y. Noguchi, Y. Murase, H. Kawaguchi, and T. Sakurai, "A large-area, flexible, and lightweight sheet image scanner," in IEEE International Electron Devices Meeting (IEDM), San Francisco, December 13-15, pp. 365-368, IEEE, 2004.

7. L. Bürgi, R. Pfeiffer, M. Mücklich, P. Metzler, M. Kiy, and C. Winnewisser, "Optical proximity and touch sensors based on monolithically integrated polymer photodiodes and polymer leds," Organic Electronics 7(2), pp. 114-120, 2006.

8. D. Mühlbacher, M. Scharber, M. Morana, Z. Zhu, D. Waller, R. Gaudiana, and C. Brabec, "High photovoltaic performance of a low-bandgap polymer," Adv. Mater. 18(22), p. 2931, 2006.

9. L. H. Slooff, S. C. Veenstra, J. M. Kroon, D. J. D. Moet, J. Sweelssen, and M. M. Koetse, "Determining the internal quantum efficiency of highly efficient polymer solar cells through optical modeling," Appl. Phys. Lett. 90(14), p. 143506, 2007.

10. J. Y. Kim, K. Lee, N. E. Coates, D. Moses, T.-Q. Nguyen, M. Dante, and A. J. Heeger, "Efficient tandem polymer solar cells fabricated by all-solution processing," Science 317(5835), pp. 222-225, 2007.

11. J. Gilot, M. M. Wienk, and R. A. J. Janssen, "Double and triple junction polymer solar cells processed from solution," Appl. Phys. Lett. 90, p. 143512, 2007.

12. A. J. Moulé and K. Meerholz, "Minimizing optical losses in bulk heterojunction polymer solar cells," Appl. Phys. B 86(4), pp. 721-727, 2007. 
13. G. Janssen, A. Aguirre, E. Goovaerts, P. Vanlaeke, P. J., and J. Manca, "Optimization of morphology of P3HT/PCBM films for organic solar cells: effects of thermal treatments and spin coating solvents," Eur. Phys. J. Appl. Phys. 37, pp. 287-290, 2007.

14. A. Llobera, V. Seidemann, J. A. Plaza, V. J. Cadarso, and S. Büttgenbach, "Integrated polymer optical accelerometer," IEEE Photonics Techn. Lett. 17(6), pp. 1262-1264, 2005. 